\title{
Intense and efficient ultraviolet electroluminescence from organic light-emitting devices with fluorinated copper phthalocyanine as hole injection layer
}

Musubu Ichikawa*, Kana Kobayashi, Toshiki Koyama and Yoshio Taniguchi

Department of Functional Polymer Science, Faculty of Textile Science and technology, Shinshu University, 3-15-1 Tokita, Ueda 386-8567, Japan

(Received

)

Abstract: We demonstrated intense and efficient ultraviolet (UV) electroluminescence (EL) from organic light-emitting devices using an oxadiazole compound (OXD-7) as an electron transporting UV light-emitting material, whose device structure is indium-tin-oxide $/ \mathrm{F}_{16} \mathrm{CuPc} / \mathrm{CBP} / \mathrm{OXD}-7 / \mathrm{LiF} / \mathrm{Al}$. Using the perfluorinated copper phthalocyanine $\left(\mathrm{F}_{16} \mathrm{CuPc}\right)$ as a hole injection material (HIM) and a carbazole derivative (CBP: 4,4'-dicarbazolyl biphenyl) as a hole transporting material with no exciplex formation nature with OXD-7 enhanced the device performances. The turn-on voltage was $10.5 \mathrm{~V}$, the maximum external quantum efficiency reached $0.8 \%$ at a current density of approximately $50 \mathrm{~mA} / \mathrm{cm}^{2}$, and the maximum radiance of EL light reached $10 \mathrm{~mW} / \mathrm{cm}^{2}$. The fluorinated $\mathrm{CuPc}$ is a potential HIM for wide-bandgap organic semiconducting material devices.

Keywords: Organic Light-Emitting Diodes, Ultra-Violet Light, Fluorinate Copper Phthalocyanine, Electroluminescence

*Corresponding author: musubu@shinshu-u.ac.jp 
Since the ground-breaking work of Tang and VanSlyke in electroluminescence (EL) from organic light-emitting diodes (OLEDs)[1], research on OLEDs has been booming and remarkable progress has been made in the last decade. OLEDs are of great interest because of their potential applications for making low-cost flat panel display products. Continuous efforts, including the development of efficient materials and ingenious device fabrications, have been carried out, leading to the highest quantum conversion efficiency (electron to photon) of nearly 100\% being reported using organometallic phosphors such as Ir complexes. [2]

In the research stream, organic semiconductors with a wide bandgap are becoming important because triplet excitons must be confined in the phosphors. In particular, wide bandgap organic semiconducting materials are attractive as blue light-emitting phosphors[3-5], because the well-known energy gap between the lowest triplet excited state $\left(T_{1}\right)$ and ground state $\left(S_{0}\right)$ of organic materials has become much smaller than that between the lowest singlet excited state $\left(\mathrm{S}_{1}\right)$ and $\mathrm{S}_{0}$. Furthermore, since wide bandgap organic semiconducting materials are potentially applicable to ultraviolet (UV: below the wavelength of $380 \mathrm{~nm}$ ) light emission devices, surface UV light-emitting devices, which are hardly available using other light-emitting technologies, could open another application field for organic devices. However, there have been only a few reports on UV OLEDs[6, 7] and deep violet OLEDs[8-10]. One reason for the scarcity of reports is that it is difficult to inject both charge carriers (electrons and holes) into wide bandgap organic semiconductors. Since organic materials work as semiconductors based on charge injection from electrodes, the carrier injection difficulties are critical. In this paper, we report efficient and bright UV OLEDs based on stable bipolar injection into wide bandgap UV light-emitting oxadiazole using a fluorinated phthalocyanine as a hole injection material.

All of the OLEDs used in this study were prepared by thermal evaporation technique onto a conventional indium tin oxide (ITO) coated glass substrate under vacuum (below $8 \times 10^{-4} \mathrm{~Pa}$ ). The substrate was treated with $\mathrm{O}_{2}$ plasma in advance. The current density $(J)$ versus applied voltage $(V)$ characteristics of OLEDs were measured with a source measurement unit (Advantest, R6245), and EL intensities were measured simultaneously with $J-V$ using a calibrated Si photodiode (Hamamatsu Photonics, S1237-1010BQ). PL and EL spectra were obtained with a spectrograph composed of a 
polychromator (Solar TII, MS7504) and a charge coupled camera (Andor, DV420-OE), calibrated with a tungsten halogen lamp. The highest occupied molecular orbital (HOMO) levels were determined from each neat thin film with Riken Keiki AC-2 or AC-3 photoelectron emission spectrometers and the lowest unoccupied molecular orbital (LUMO) levels were estimated from HOMO levels and band gaps determined by the spectral edge of the UV-visible absorption spectrum of each film. All chemicals were purified by vacuum sublimation before use.

While oxadiazoles (OXDs) are well known as good electron transporting materials[11], they are, by nature, also well known as efficient light emitters for scintillation counters. Moreover, since the oxadiazole ring restricts extensions of $\pi$-conjugation beyond the ring, OXDs often emit UV fluorescence. For all of these reasons, OXDs can be used as light-emitting electron transporting materials for UV OLEDs. While OXD-7 depicted in Figure 1 is an efficient electron transporting material reported by Hamada et al.[12], since the meta connection through the central phenylene strongly imposes restrictions on $\pi$-conjugation extension, OXD-7 emits UV light as a fluorescence as shown in Figure 2a. Therefore, OXD-7 will be valuable for an electron transporting UV emitter for UV OLEDs.

As shown in the energy diagram of Fig. 1, the HOMO level of OXD-7 is so deep that it is difficult to attain stable and efficient bipolar (especially, hole) injection into OXD-7 to get UV light based on charge recombination. Therefore, enhancing hole injection is the top priority. Figure 3a shows $J-V$ of an ITO/NPB(50 nm)/OXD-7(50 $\mathrm{nm}) / \operatorname{LiF}(0.5 \quad \mathrm{~nm}) / \mathrm{Al}$ device, where $\mathrm{NPB}$ N,N'-di-naphthalen-1-yl-N,N'-diphenyl-benzidine. As shown in the figure, this OLED was hard to operate and it broke down easily, as a result of the difficulty of hole injection from a conventional hole transporting material (HTM), NPB, to the OXD-7 layer because of the large energy barrier for hole injection at the NPB/OXD-7 interface. In addition, the OLED showed sky-blue electroluminescence as shown in Fig 2c resulting from exciplex formation at the interface because NPB exhibited a rather strong electron-donating property. Furthermore, a single-layer device (ITO/OXD-7/LiF/Al) could flow current at a lower voltage than the double-layer device but the single-layer device emitted no light. 
OXD-7 demands HTMs with a deep HOMO level to avoid the formation of exciplexes at the HTL/OXD-7 interface to attain high quantum efficiencies of EL and lower operating voltages. Carbazoles are well-known HTLs, and their HOMO levels are usually deeper than those of conventional HTMs such as NPB, N,N'-diphenyl-N,N'-di(m-tolyl)benzidine, and other arylamine derivatives. However, using a deep HOMO HTM causes a large energy barrier for hole injection at the ITO/HTM interface. Therefore, we used perfluorinated copper phthalocyanine[13] $\left(\mathrm{F}_{16} \mathrm{CuPc}\right)$ as a hole injection material (HIM). Phthalocyanines are conventionally used as HIMs, and fluorination reduces the HOMO and LUMO levels. Therefore, we expected that $\mathrm{F}_{16} \mathrm{CuPc}$ would work as an efficient HIM between ITO and carbazole HTMs. Figure 1 shows the energy diagram of $\mathrm{F}_{16} \mathrm{CuPc}$ and 4,4'-dicarbazolyl biphenyl (CBP) as a representative carbazole HTM. Good device performance can be expected the triple-layer device.

Figure 3a shows $J$ - $V$ characteristics of the triple-layer device: $\mathrm{F}_{16} \mathrm{CuPc}(20$ $\mathrm{nm}) / \mathrm{CBP}(30 \mathrm{~nm}) / \mathrm{OXD}-7(50 \mathrm{~nm})$, together with $J-V$ characteristics of the CBP (50 $\mathrm{nm}) / \mathrm{OXD}-7(50 \mathrm{~nm})$ device, and Figure 3b shows EL intensity and external quantum efficiency vs. current density characteristics of this triple-layer device. As we can see from the figures, the device could be operated at a much lower voltage than the NPB/OXD-7 and CBP/OXD-7 double-layer devices. It exhibited a practical operation performance, and the turn-on voltage, which is defined here as the voltage to attain an electroluminescence power density of $1 \mu \mathrm{W} / \mathrm{cm}^{2}$, was $10.5 \mathrm{~V}$. The device's external quantum efficiency increased with its operating current density up to $50 \mathrm{~mA} / \mathrm{cm}^{2}$, and then decreased with increasing operating current density. The maximum efficiency reached $0.82 \%$ at a current density of $50 \mathrm{~mA} / \mathrm{cm}^{2}$, and the efficiency at an EL power density of $5 \mathrm{~mW} / \mathrm{cm}^{2}$ was approximately $0.75 \%$. The maximum external power density of EL light of the optimum device reached $10 \mathrm{~mW} / \mathrm{cm}^{2}$. Hence, we concluded that stable and efficient bipolar operation was attained.

Figure 2c shows the normalized EL spectra from the $\mathrm{F}_{16} \mathrm{CuPc} / \mathrm{CBP} / \mathrm{OXD}-7$ device. As shown in the figure EL spectra from this device exhibited no dependence on excitation current densities. The EL spectra peaked at a wavelength of $372 \mathrm{~nm}$, which is definitely in the UV region below $380 \mathrm{~nm}$. Therefore, we successfully obtained UV light emission from OLEDs. Although there have been a few reports on UV OLEDs, the 
peak wavelength of $372 \mathrm{~nm}$ is among the shortest peak wavelength reported in the literatures on OLEDs.[6-10]

As we can see from Figure 2c, the EL spectrum exhibits some differences from the photoluminescence (PL) spectrum of OXD-7. The differences probably result from optical absorption by $\mathrm{F}_{16} \mathrm{CuPc}, \mathrm{CBP}$, and the ITO substrate. Figure $2 \mathrm{~b}$ also plots the transmittance spectra of these layers and the substrate. As shown in the figure, unfortunately, the $\mathrm{F}_{16} \mathrm{CuPc}$ layer absorbed emitted luminescence, and the absorption was strong at around $355 \mathrm{~nm}$, corresponding to the highest energy vibronic band of PL of OXD-7. Hence, we reasonably conclude that there was a recombination area in the OXD-7 layer and that stable and efficient bipolar injection into OXD-7 was achieved.

The absorption loss of each layer and of the substrate can be estimated from overlap integrals between the PL spectrum of OXD-7 and each transmittance spectrum. The estimated losses were $20 \%$ for the $\mathrm{F}_{16} \mathrm{CuPc}$ layer, $18 \%$ for the substrate, and $7 \%$ for the CBP layer, respectively, which leaves only 55\% luminescence. If we can reduce the optical absorption losses, we can gain higher external quantum efficiency up to $1.5 \%$ and more intense radiance up to $18 \mathrm{~mW} / \mathrm{cm}^{2}$.

In conclusion, we demonstrated intense and efficient UV electroluminescence from OLEDs, with the structure of $\mathrm{ITO} / \mathrm{F}_{16} \mathrm{CuPc} / \mathrm{CBP} / \mathrm{OXD}-7 / \mathrm{LiF} / \mathrm{Al}$. Using $\mathrm{F}_{16} \mathrm{CuPc}$ as an HIM led to a device that worked well: the turn on voltage was $10.5 \mathrm{~V}$, the maximum external quantum efficiency reached $0.82 \%$ at a current density of 50 $\mathrm{mA} / \mathrm{cm}^{2}$, and the maximum radiance of EL light reached $10 \mathrm{~mW} / \mathrm{cm}^{2}$. Thus, fluorinated $\mathrm{CuPc}$ is a potential hole-injection material for wide bandgap organic semiconducting material devices.

The authors thank Dr. Y. Nakajima and Mr. D. Yamashita of Riken Keiki for measuring the ionization potential of deep HOMO compounds with their special photoelectron spectroscopic equipment (Riken Keiki AC-3). This work was supported by the Cooperative Link for Unique Science and Technology for Economy Revitalization (CLUSTER) of Japan's Ministry of Education, Culture, Sports, Science and Technology. It was also supported by the Ministry’s 21st Century COE program. 


\section{References}

[1] C. W. Tang, S. A. VanSlyke, Appl. Phys. Lett. 51 (1987) 913.

[2] C. Adachi, M. A. Baldo, M. E. Thompson, S. R. Forrest, J. Appl. Phys. 90 (2001) 5048.

[3] R. J. Holmes, S. R. Forrest, Y.-J. Tung, R. C. Kwong, J. J. Brown, S. Garon, M. E. Thompson, Appl. Phys. Lett. 82 (2003) 2422.

[4] S. Tokito, T. Iijima, Y. Suzuri, H. Kita, T. Tsuzuki, F. Sato, Appl. Phys. Lett. 83 (2003) 569.

[5] R. J. Holmes, B. W. D'Andrade, S. R. Forrest, J. L. X. Ren, M. E. Thompson, Appl. Phys. Lett. 83 (2003) 3818.

[6] T.-C. Chao, Y.-T. Lin, C.-Y. Yang, T. S. Hung, H.-C. Chou, C.-C. Wu, K.-T. Wong, Adv. Mater. 17 (2005) 992.

[7] L. Zou, V. Savvate'ev, J. Booher, C.-H. Kim, J. Shinar, Appl. Phys. Lett. 79 (2001) 2282.

[8] C.-H. Yuan, S. Hoshino, S. Toyoda, H. Suzuki, M. Fujiki, N. Matsumoto, Appl. Phys. Lett. 71 (1997) 3326.

[9] C. F. Qiu, L. D. Wang, H. Y. Chen, M. Wong, H. S. Kwok, Appl. Phys. Lett. 79 (2001) 2276.

[10] K. Okumoto, Y. Shirota, Appl. Phys. Lett. 79 (2001) 1231.

[11] H. Tokuhisa, M. Era, T. Tsutsui, S. Saito, Appl. Phys. Lett. 66 (1995) 3433.

[12] Y. Hamada, C. Adachi, T. Tsutsui, S. Saito, Jpn. J. Appl. Phys. 31 (1992) 1812.

[13] Z. Bao, A. J. Lovinger, J. Brown, J. Am. Chem. Soc. 120 (1998) 207. 


\section{Figure Captions}

Figure 1. Schematic diagram and device structure of the device together with chemicals used. Here, the work function of $\mathrm{LiF} / \mathrm{Al}$ cathode is assumed to be same as that of lithium metal.

Figure 2. a: PL spectrum of OXD-7, b: transmittance spectra of the layers ( $\mathrm{F}_{16} \mathrm{CuPc}$ and $\mathrm{CBP}$ ) and the ITO substrate, c: normalized EL spectra from the triple-layer device $\left(\mathrm{F}_{16} \mathrm{CuPc} / \mathrm{CBP} / \mathrm{OXD}-7\right)$ and double-layer device (NPB/OXD-7). Figure 3c showed EL spectra from the triple-layer device at different current densities of 7.5 and $125 \mathrm{~mA} / \mathrm{cm}^{2}$.

Figure 3. a: Current density vs. voltage characteristics of the double-layer and triple-layer devices. b: EL intensity and external quantum efficiency vs. current density characteristics of the triple-layer device. 

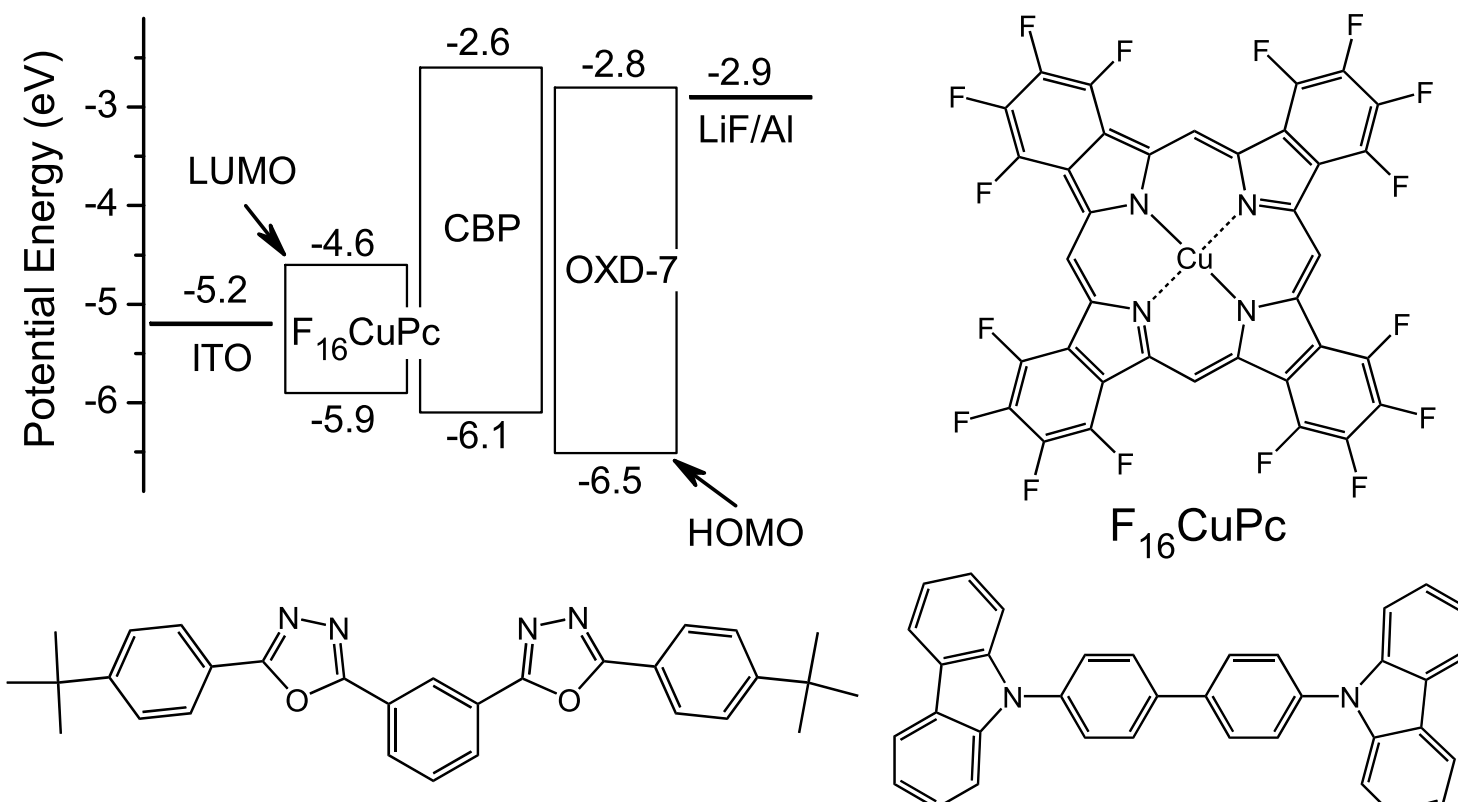

OXD-7

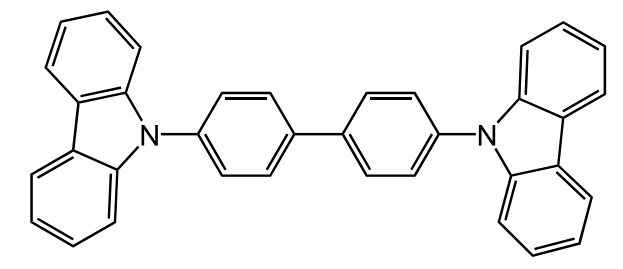

CBP

Figure 1 


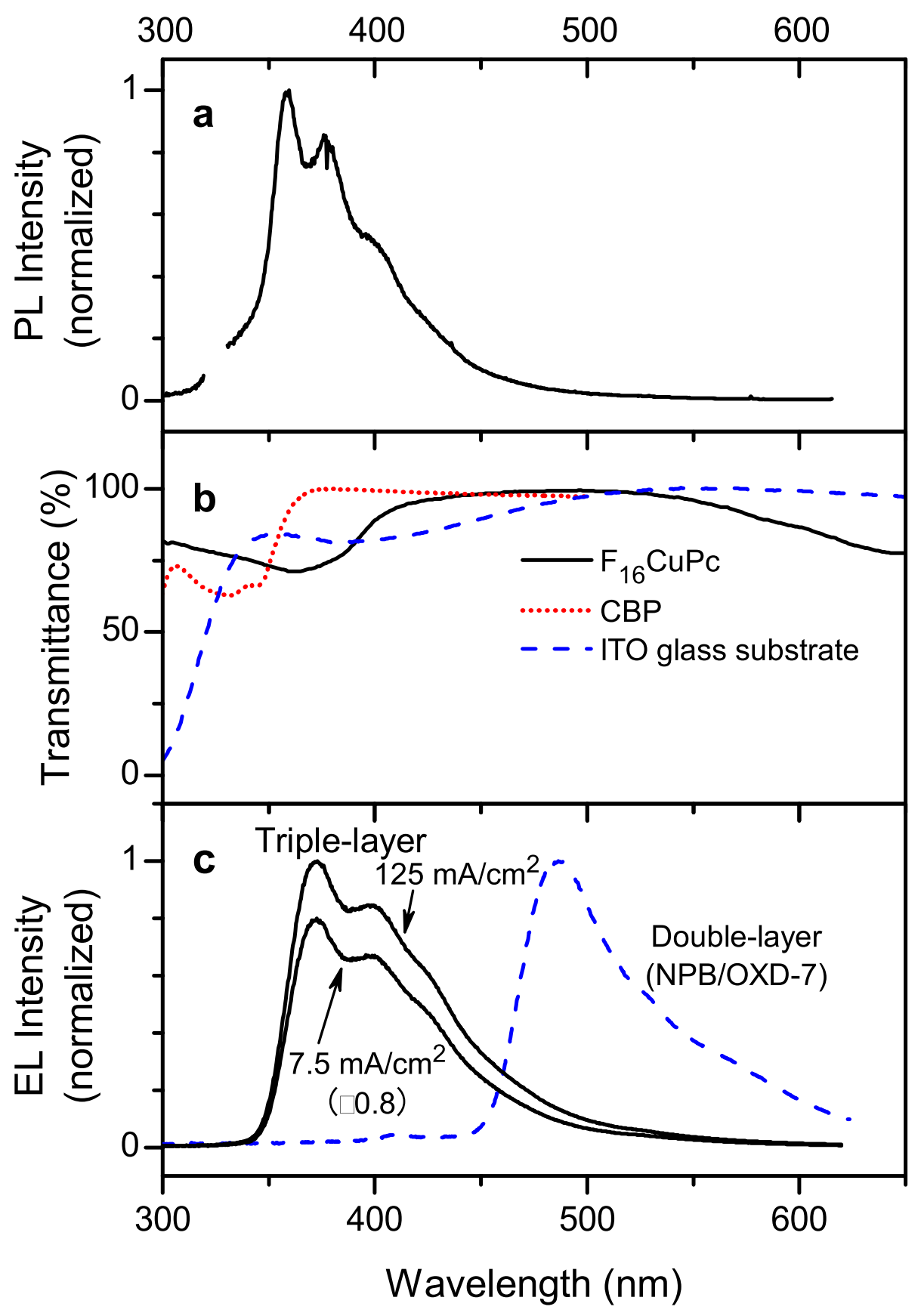

Figure 2 

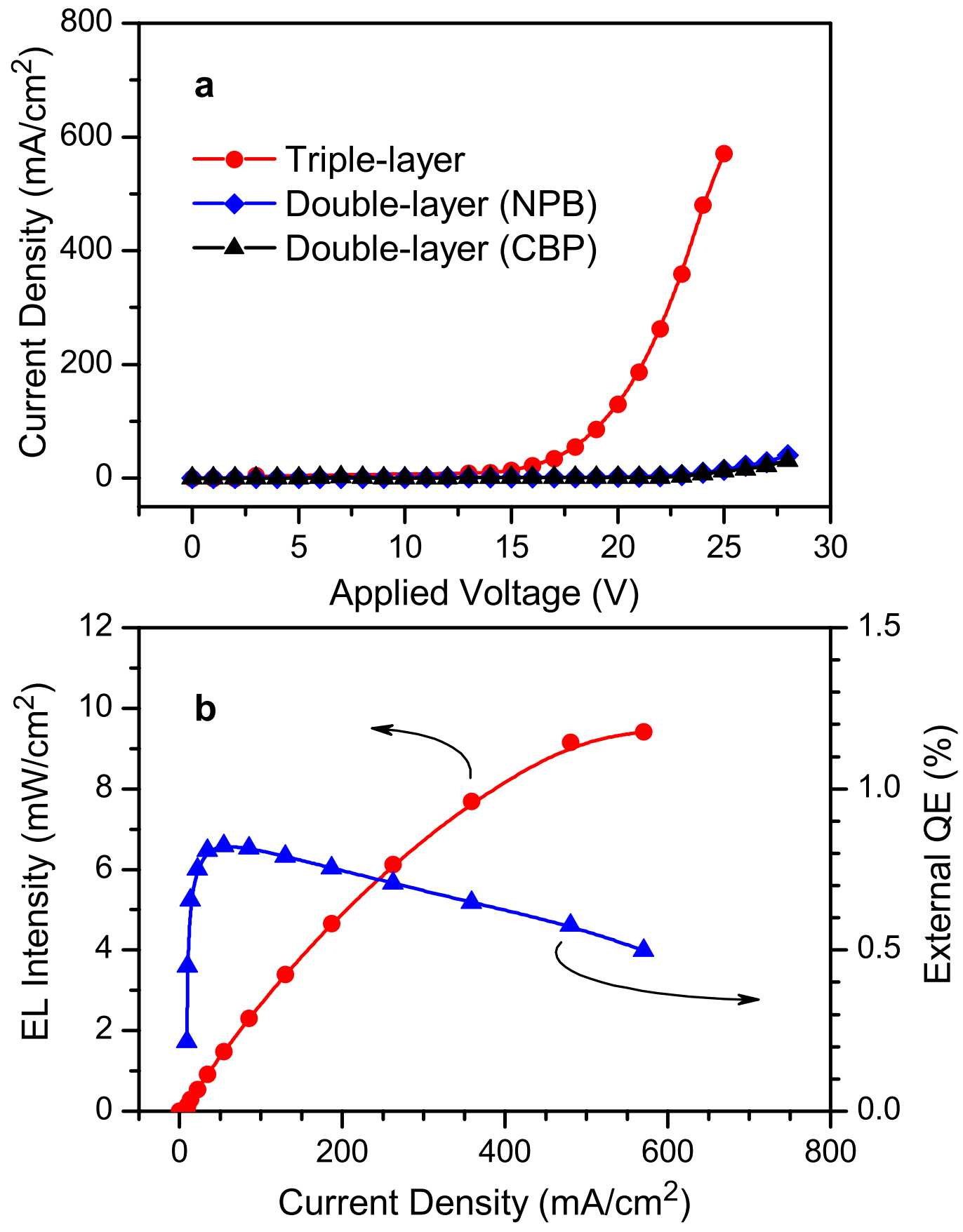

Figure 3 